\title{
Development of a Conceptual Model of Disease Progression for Use in Economic Modeling of Chronic Obstructive Pulmonary Disease
}

\author{
Maggie Tabberer, MSc ${ }^{*}$, Sebastian Gonzalez-McQuire, MSc, Hana Muellerova, PhD, Andrew Briggs, \\ DPhil, Maureen Rutten-van Molken, PhD, Mike Chambers, MSc, David A. Lomas, PhD FRCP
}

Value Evidence and Outcomes, GSK R\&D, Stockley Park, UK (MT); Formerly Global Health Outcomes, GSK R\&D, Stockley Park, UK (SGM); Worldwide Epidemiology, GSK R\&D, Stockley Park, UK (HM); Institute of Health and Wellbeing, University of Glasgow, Glasgow, UK (AB); ICON Health Economics, Morristown, NJ, USA (AB); Institute for Medical Technology Assessment, Erasmus University/Erasmus Medical Centre, Rotterdam, The Netherlands (MRvM); Global Market Access and Healthcare Solutions, GSK, Brentford, UK (MC); Wolfson Institute for Biomedical Research, University College London, London, UK (DAL).

\section{*Address correspondence to:}

Maggie Tabberer

Director, Respiratory Patient Focused Outcomes, Value Evidence and Outcomes, GSK

Stockley Park West (B10), Uxbridge, Middlesex UB11 1BU, United Kingdom

Tel: +44 208990 2041; Fax: +44 2089903505

Email: margaret.x.tabberer@gsk.com

Funding: This study was funded by GSK.

This work has previously been published in abstract form and was presented in two parts at the International Society for Pharmacoeconomics and Outcomes Research - 14th Annual European Congress 2011 (literature review) and at the International Society for Pharmacoeconomics and Outcomes Research - 15th Annual European Congress 2012 (conceptual model development). 
Key words: Conceptual model, chronic obstructive pulmonary disease (COPD), literature review, Delphi Panel, economic modeling

Running Title: COPD conceptual model for economic modeling

Abbreviated Title: Development of a conceptual model of COPD for economic modelling

Word count: 3934

Financial support for this study was provided entirely by GSK. The funding agreement ensured the authors'

independence in designing the study, interpreting the data, writing, and publishing the report. The following authors are employed by the sponsor: MT, HM, MC; SGM is a former employee of the sponsor. 


\section{ABSTRACT}

Background. To develop and validate a new conceptual model (CM) of chronic obstructive pulmonary disease (COPD), for use in disease progression and economic modeling. The CM identifies and describes qualitative associations between disease attributes, progression and outcomes.

Methods. A literature review was performed to identify any prior published CM or literature reporting on the patient impact and association of COPD disease attributes with outcomes. After critical analysis of the literature, a Steering Group of experts from the disciplines of health economics, epidemiology and clinical medicine was convened to develop a draft CM, which was refined using a Delphi process. The refined CM was validated by testing for associations between attributes using data from the Evaluation of COPD Longitudinally to Identify Predictive Surrogate Endpoints (ECLIPSE).

Results. Disease progression attributes included in the final CM were history and occurrence of exacerbations, lung function, exercise capacity, signs and symptoms (cough, sputum, dyspnea), cardiovascular disease comorbidities, 'other' comorbidities (including depression), body composition (body mass index), fibrinogen as a biomarker, smoking, and demographic characteristics (age, gender). Mortality and health related quality of life were determined to be the most relevant final outcome measures for this model, intended to be the foundation of an economic model of COPD.

Conclusion. The CM is being used as the foundation for developing a new COPD disease progression model. The CM provides a framework for analysis of patient level data and is available as a reference for the implementation of further disease progression and economic models. 


\section{INTRODUCTION}

Disease progression and economic modeling of healthcare interventions, particularly pharmaceutical technologies, has traditionally focused on the estimation of costs and benefits, employing measures of efficacy collected during clinical trials or observational studies. In chronic obstructive pulmonary disease (COPD), disease progression and economic models have mainly focused on lung function and exacerbations as the main attributes of the disease. Although these are relevant measures of disease severity and progression, research has demonstrated that COPD is a systemic disease with multiple attributes that impact on disease progression and health outcomes [1]. The development of new therapeutic interventions for COPD is increasingly focused on these systemic effects, which may be associated with simultaneous changes in several intermediate outcomes, with potential for differential efficacy in different COPD phenotypes. Consequently, any new disease progression or economic model developed for COPD to support such interventions should take into consideration multiple aspects of the disease, both symptoms and health related quality of life experienced by the patient and those that affect clinical progression.

A recent update of guidelines for good economic modeling research practice recommended the development of a conceptual model (CM) as a basis for model development [2]. A CM representing a particular disease should ideally illustrate the relationship between determinant aspects of the disease and its outcomes that can be identified and measured, thereby providing a framework that highlights potential interactions between attributes and helps the researcher to avoid any 'double counting' of effects in the subsequent economic model. A CM also helps to illustrate how a healthcare intervention which may be directed at changing one aspect of the disease may have an indirect impact on other aspects of the disease that have not been measured in clinical trials.

The objective of the present study was to develop a CM for use in modeling disease progression and subsequently to underpin an economic model (to be reported separately) for the assessment of cost effectiveness of therapies in COPD. The CM identifies and describes relationships between disease attributes, disease progression and health outcomes. These relationships were then tested using existing data from observational and clinical cohorts to ensure only relevant measurable attributes were included in the final CM (the CM was limited to those variables reported in these cohorts in order to facilitate validation of the $\mathrm{CM}$ ). 


\section{METHODS}

Literature review. We conducted a systematic literature search to identify publications of CMs in COPD, including those represented in economic models, to identify studies reporting the associations between disease attributes and their prognostic value in terms of both disease progression and health outcomes. The term 'attribute' is used here to collectively refer to any determinant, endpoint or health outcome associated with the measurement of COPD. The attributes of interest for the purposes of this study are those that may affect health status (health-related quality of life) and mortality, either directly or by influencing an intermediate outcome such as the frequency of exacerbations.

The literature search was conducted using MEDLINE and EMBASE, and was limited to articles published in the English language from 2000 to 2012. Publications earlier than 2000 were excluded to reflect more recent advances in disease understanding. The search strategy was designed to identify studies reporting CMs and relationships, associations (correlations and/or regression analyses) with COPD endpoints and disease progression. The terms used in the literature search included: conceptual model, regression model, associations, COPD, endpoint model, correlation, conceptual framework, relationships, attributes, endpoints and outcomes. The review included studies presenting results of associations between COPD disease attributes and health outcomes. Attributes were identified that either directly affect the course of the disease or that modify other 'intermediate' attributes that have prognostic value. Clinical trials specifically designed to report the efficacy and/or effectiveness of therapeutic interventions (pharmacologic treatments, pulmonary rehabilitation etc.), were excluded from the results of in this search. Such trials are not designed primarily to report on the relationships between disease attributes and health outcomes, and where this occurs the selection of attributes and outcomes is related to the effects of the intervention studied. Study design features (including duration of intervention, length of follow up and, particularly, inclusion and exclusion criteria) also mean that any associations reported may not be applicable to the broader disease concept of COPD over the course of the disease. Specifically, the aim of the review was to support development of a CM as an underlying structure for an economic model of COPD in which the effects of different interventions would be incorporated by applying (different) treatment effects to the baseline or 'untreated' disease progression model. 
Whilst CMs relating to specific interventions have been developed (e.g. those aimed at the treatment of dyspnea), these include limited attributes and components related to the specific intervention.

Steering Group and Delphi Panel Two groups were convened to guide the development of the CM. The first was a project Steering Group consisting of experts in health economics, epidemiology, and clinical medicine. Members of the Steering Group, all of whom are experienced COPD researchers and economic modellers, developed a draft CM of COPD (based on the literature review and informed by their own expertise) for testing by the second group, convened as a Delphi Panel. The role of the Delphi Panel was to refine the initial CM drawn up by the Steering Group. The Steering Group provided oversight of model development, including quantification and validation of associations between attributes, and consequently guided development of the disease progression and economic model. The members of both the Steering Group and the Delphi Panel were reimbursed for time spent completing the questionnaires and participating in the panel.

Delphi methodology is a recognized method for gaining expert consensus and has been used extensively across the social sciences [3-5]. It is useful for gaining the input of a variety of experts in a timely and efficient manner through a series of questionnaires and feedback; two or three rounds of questionnaires are usually required to gain consensus. The method encourages equal participation of all panelists and removes some negative aspects of group dynamics from the process. Five to eight panelists are suggested as optimal. In this study, five academics with a wide range of clinical and epidemiological experience in COPD were invited to participate. Panel members were selected to ensure that all relevant aspects of COPD from the literature review and from personal expertise were identified in the final CM.

A Delphi Panel exercise using modified methodology was conducted by email between 23 January 2012 and 15 February 2012. Two rounds of questionnaires were conducted to gain consensus. In round one, panel members were sent the draft $\mathrm{CM}$ and an initial questionnaire with instructions for individual completion. The information returned by panel members was then tabulated and collated. A second questionnaire (round two), following the same general format of the first, was circulated to panel members together with the anonymized, collated results of round one, and the individual panel member's previous responses to each question. Personalized supplementary questions were also included where needed, to seek clarity on responses from round one. 
The questionnaires consisted of a number of questions related to the draft $\mathrm{CM}$ and included graphical breakdown of sections of the $\mathrm{CM}$ for simplification and to allow for systematic analysis of the different attributes of COPD. Panel members were asked to perform the following tasks: (1) assess the disease attributes and concepts included in the draft CM for completeness; (2) suggest additional attributes that might be missing; (3) evaluate the relationships between attributes and the direction of those relationships; (4) qualitatively estimate the strength of the relationships (high/medium/low) to indicate which attributes were likely to be essential for quantifying disease progression and health outcomes; (5) suggest additional relationships and/or directional links that might be missing; and (6) recommend the most appropriate measure for each attribute.

The graphical breakdown of the draft CM comprised sections on body composition, comorbidities, exacerbations, lung function, exercise capacity, signs and symptoms, triggers, depression, fibrinogen, and demographic and other characteristics. For each section, panelists were also asked to comment on the proposed measurement metrics for the attribute and suggest other preferred measures.

Associations between attributes. Disease attributes in the CM were divided into two categories: baseline attributes (those that do not change over time, change independently of disease progression, or for which the impact on disease progression is constant over time), and disease progression attributes (those for which impact on disease progression changes over time).

To test the two levels of association, we first implemented a pairwise analysis using baseline variables to explore strength and direction of effect among all disease progression attributes identified in the CM (lung function, exacerbations, signs and symptoms, and exercise capacity). We then evaluated the pairwise associations to evaluate inter-dependent associations over time, and applied random coefficient models to evaluate endogeneity and the potential for non-linearity, with an appropriate distribution to establish the strength of the association over time. For both analyses we used data from the Evaluation of COPD Longitudinally to Identify Predictive Surrogate Endpoints (ECLIPSE) study [6,7]. The ECLIPSE population comprised 2164 clinically stable COPD patients, 337 smokers with normal lung function and 245 never smokers; only data from the COPD patients was considered during the development of the CM. At baseline, COPD patients were older, had more smoking pack years, were more symptomatic (as reported by the modified Medical Research Council Questionnaire [mMRC] and the COPD- 
specific version of the St. George's Respiratory Questionnaire [SGRQ-C]), had more comorbidities, and showed airflow limitation compared with smokers with normal lung function and never smokers.

Significance testing of associations between attributes and of attributes with final health outcomes both at baseline (to establish association between attributes) and longitudinally (to establish associations with changes over time in attributes) supported their categorization, and confirmed which attributes could be fully quantified in the subsequent disease progression model. The CM was thereby designed to guide the development of a disease progression model.

The estimated associations and direction of effect were assessed by the Steering Group for clinical plausibility. To assess the strength of effect, both at baseline and over time, the exacerbation risk equations used the Wald chi-square test; other risk equations used the t-test. If the $P$-value was $\geq 0.05$, the association was deemed nonstatistically significant. Associations between attributes postulated in the draft CM were excluded from the final CM if no significant association was seen when the relationships were tested against the ECLIPSE data.

Attributes categorized as baseline within the model were: body composition (body mass index, BMI), cardiovascular disease (CVD) comorbidity, depression, fibrinogen, smoking, exacerbation history and demographic characteristics. Disease progression attributes were: exacerbations, symptoms, exercise capacity and lung function . Final health outcomes defining the health status of the patient were mortality and health related quality of life.

Final conceptual model. The CM was then refined based on the results of the Delphi consultation, available data and confirmation of associations at baseline and longitudinally. The Steering Group assessed whether there were sufficient data available to quantify the effects of associations prior to finalizing the CM; this pragmatic approach was taken to ensure that the final CM was fit for its intended purpose as a basis for economic modeling.

Role of the funding source. The literature review was performed by SGM, who was an employee of GSK at the time. The Steering Group and Delphi Panel members were chosen and convened by MT, HM, MC (all current employees of GSK) and SGM, and members entered into a contract with GSK. The Delphi Panel questionnaire was developed by MT. Steering Committee meetings were virtually hosted by GSK. 


\section{RESULTS}

Literature review. The MEDLINE and EMBASE database searches yielded a total of 443 citations. After review of abstracts, 274 of the 443 citations were rejected. Of the 169 full-text articles retrieved for potential inclusion, 125 were excluded during the second-level selection process. Thus, a total of 44 published papers were identified that reported CMs and/or associations between attributes and health outcomes in COPD. Figure 1 depicts the article flowchart through the study selection process, and reasons for article exclusion.

Attributes and health outcomes were categorized as dependent or explanatory according to the distinction made in the source literature between 'cause' (explanatory; e.g. exacerbations) and 'effect' (dependent; e.g. worse lung function). The frequency with which each was reported was then used to identify associations and their directionality (Figure 2). The count reflects the number of times a particular disease attribute was reported in the literature as being associated with either another attribute or a health outcome of COPD. A total of seven studies reporting CMs of COPD, which also reported associations between attributes, and 37 studies reporting at least one association between endpoints and disease progression were found in the literature. Of the seven CMs examined, six described a single aspect of COPD: cognitive function [25], dyspnea [24], brain function [8], design of patient related interventions [17], activity [14] and functional performance [26]. Only one study [22] described a broader set of determinants of health status in COPD patients: physiological functioning, patient complaints, functional impairment and health related quality of life.

Of the 44 published papers, 31 used regression analysis to estimate associations between attributes of COPD, including symptoms (mainly dyspnea), health status, exercise, lung function, exacerbations, quality of life, biomarkers, comorbidities, mortality and healthcare utilization. The attributes identified are shown in Figure 3. Exercise capacity was reported to be associated with a variety of attributes of COPD (symptoms, health status, lung function and exacerbations), as well as with health outcomes (hospitalizations), highlighting the need to consider this attribute when conceptualizing COPD. Although some were identified in the review, interventional studies of pharmacologic treatment and pulmonary rehabilitation were excluded from the development of the CM which represents the natural history of the disease process, only observational studies were included. Interventional studies 
are usually selective in the population enrolled and typically have a limited duration of both intervention and follow up.

The literature review did not identify any CM that described the relationships between all aspects of the disease within one framework. The need to develop a CM of COPD that reflects current and emerging disease understanding, and which can support the development of a disease progression model was confirmed.

Steering Group-based draft conceptual model. The draft CM developed by the Steering Group included attributes of COPD, baseline characteristics and other factors that might influence disease progression, such as psychological and emotional factors. To simplify this draft model attributes were grouped, e.g., physical signs and symptoms of COPD were aggregated together. Hypothesized directions of associations were also included. The draft CM reflected the data available from the ECLIPSE dataset, and is shown in Figure 4a). Covariate data, available at baseline only, are shown at the top of the figure.

Delphi Panel. Although the panel did not identify any new attributes for inclusion, they did identify that the CM should split the concept of signs and symptoms of COPD shown in the draft CM and display the individual signs and symptoms separately. Dyspnea, cough and/or sputum, fatigue and signs of hyperinflation, inflammation, and hypoxia were recommended as meaningful and measurable. The panel also recommended further changes to split exacerbations into different levels of severity, and to specify CVD separately from 'other' comorbidities.

The panel proposed some additional links and recognized that the relationships between individual attributes differ in strength, with some relationships having a greater impact on disease progression health status and mortality than others (Table 1). The panel also identified that the strength of a relationship between two attributes may differ when used to describe (baseline) stratification of patients than when used to describe disease progression. Panelists also indicated where relationships existed but were considered too weak to independently drive disease progression and health outcomes.

Associations between attributes. The relationships proposed in the draft CM and modified by the Delphi Panel were tested using data from the ECLIPSE cohort.

Correlation analyses of all baseline variables confirmed the direction of effect and showed statistical significance, except for the associations between CVD comorbidities and exacerbations, and between depression and 
exacerbations (Table 1). All of the proposed associations over time between the explanatory and dependent variables were shown to be significantly associated $(P<0.0001)$ over time (e-Appendix 1$)$.

Final conceptual model. The attributes included in the final CM were: exacerbations, lung function, exercise capacity, signs/symptoms (cough, sputum, dyspnea), CVD comorbidities, 'other' comorbidities (including depression), body composition (BMI), fibrinogen as a biomarker, smoking, exacerbation history and demographic characteristics (age, gender). Mortality and health related quality of life were agreed to be the most relevant (final) health outcome measures. Some relationships and attributes in the draft CM were found difficult to quantify due to lack of prior published evidence and/or data available in the ECLIPSE cohort and were therefore excluded from the refined CM. These included infections, environmental factors, psychological/emotional factors, socioeconomic status and ethnicity.

In the final CM, exercise was identified as having an independent effect on both exacerbation frequency and lung function. Intuitively this makes sense as increased exercise (or physical activity) is likely to improve lung function [52]. Exercise might also be associated with a reduced exacerbation rate independently of any lung function effect $[53,54]$. However, rather than measuring the patient's usual physical activity over time [55] the 6-minute walk test distance (6MWD) measures exercise capacity at a point in time [56]. Although 6MWD represents a widely used and standardized measure of exercise capacity, it can be influenced by patient motivation and the expected relationship between exercise and lung function may not be demonstrated in all patient cohorts where 6MWD is measured [57]. Therefore, the directional effect of 6MWD on lung function was removed in the final CM. In the final model, the (uni-directional) associations between forced expiratory volume in 1 second $\left(\mathrm{FEV}_{1}\right)$, exacerbations and 6MWD were retained to demonstrate the influences of $\mathrm{FEV}_{1}$ and exacerbations on 6MWD. The final CM is presented in Figure $4 \mathrm{~b}$.

The final CM was then tested to validate the relationships identified using data from the TORCH study, a clinical study including optimization of therapy with a 3-year follow up designed to reflect clinical practice. Long-term observation of health-related quality of life and, particularly, mortality (the final outcomes of the CM) were possible. Further details are provided in the supplementary materials. 


\section{DISCUSSION}

Disease progression and economic models used to support economic evaluations of healthcare interventions should be based on CMs that describe the relationships between all relevant aspects of the disease and relevant health outcomes. Although there is considerable published evidence describing the associations between specific aspects of the disease, such as symptoms and health outcomes, the indirect relationships with disease progression are not addressed. No study has previously developed a CM that seeks to understand the relationship between all relevant attributes and health outcomes in the context of COPD disease progression.

The CM was developed using a two-step approach that allowed for an assessment of evidence from existing CMs to be combined with clinical expertise and other specialties (the attributes identified in the literature and by the Delphi panel). The final CM, intended to underpin our economic model of COPD was then refined using existing baseline and longitudinal COPD patient level data (from the ECLIPSE and TORCH studies) to provide a framework for understanding the factors influencing COPD disease progression and health outcomes. This methodology approach to developing a CM should ensure that relevant aspects of a disease are considered.

The literature review identified some studies reporting CMs of aspects of disease progression in COPD patients and provided a basis for the development of the draft CM by the Steering Group. It also identified the important attributes required to represent disease progression and health outcomes in COPD and highlighted the need to qualitatively characterize both COPD disease progression and health outcomes and to quantitatively test the resulting CM. Despite the breadth of the initial literature review that was conducted, relatively few $(n=44)$ publications were identified for data extraction. Of a total of 443 citations that were identified using the pre-selected range of search terms, 399 were deemed unsuitable primarily because they described clinical trials or health interventions in a proscribed sample of the overall COPD population rather than conceptual models reporting relationships between attributes, or because their primary focus was not on COPD. Interventional studies are also usually limited in duration of both intervention and follow up as well as in the attributes measured. The intervention studied will drive both the attributes included and the population studied; for example clinical trials of bronchodilator therapies typically recruit symptomatic patients with limited or no history of COPD exacerbations 
who are not representative of the total COPD population. Although the relative lack of suitable publications somewhat hindered the development and validation of the CM described here and can therefore be considered a limitation, it may also reflect that there exist unmet needs in the economic modeling of COPD disease progression.

The use of a modified Delphi approach refined the draft CM by adding more parameters into the model and estimating directions and strength of associations based on clinical experience. The panel endorsed most of the attributes proposed by the Steering Group in the draft CM and contributed significantly to the decisions made around inclusion and exclusion of parameters and direction of associations reflected in the final CM.

The revised draft model, following input from the Delphi Panel, provided a detailed picture of all possible attributes and associations describing COPD disease progression. However, implementation of such a comprehensive $\mathrm{CM}$ as a disease progression model for economic evaluation requires large amounts of data, which may be challenging to obtain and analyze. The approach used to validate the associations between attributes and implement the revised draft of the $\mathrm{CM}$ was, by necessity, pragmatic, in that it was informed by the availability of evidence to support a quantitative estimation of the associations and correlations proposed by the Delphi Panel. The use of patient-level data from the ECLIPSE study was essential in permitting a quantification of the relationships within the CM. This dataset includes measures of the majority of the attributes identified, with the exception of some socio-economic and environmental factors.

Quantification of the associations described in the CM may also be limited by the initial use of a single cohort of patients recruited in secondary care (ECLIPSE) to determine the relative strength and direction of relationships between attributes. The ECLIPSE cohort was used as the only large-scale study in which all of the attributes included in the analysis were measured, and in which the follow-up period was of sufficient duration for health outcome data to be recorded. Exploration of the associations between the selected attributes using pairwise correlation showed that most were statistically significant. Although statistical significance of these correlations could be expected based on the relatively large size of the ECLIPSE study we believe that the selected attributes do impact patients' disease progression in COPD. The current study only assessed the existence of longitudinal associations between the disease progression attributes (lung function, exacerbation, symptoms and exercise capacity). A further limitation is that ECLIPSE did not include data that enabled an assessment of the impact of patient knowledge, attitude, and skills (such as illness perceptions, coping behavior, and self-management abilities) 
to affect disease progression. Further research estimating relationships between baseline parameters and their longitudinal correlation with other attributes would improve understanding of how best to use these parameters in disease modeling.

The strength of the present CM lies in the thorough and stepwise approach to its development, starting with a broad perspective of the disease, where all potential associations between attributes were considered, and only then focusing the $\mathrm{CM}$ to reflect the underlying purpose for its development as a basis for economic modeling of the disease. Validation of the draft CM using clinical experts, health economists and data from the ECLIPSE study resulted in a robust and comprehensive $\mathrm{CM}$ that can guide future disease modeling. For practical reasons, patients and caregivers were not included in our development of the CM: their involvement in further validation exercises is desirable.

To date, conceptual modeling to support health economic evaluation has not been widely used in a formal sense. However, interest in the formal use of CMs to support health service decision making is growing. The recently reported joint Society for Medical Decision Making (SMDM)/ International Society for Pharmacoeconomics and Outcomes Research (ISPOR) Task Force on modeling devoted a whole paper to conceptualizing a model [2] and the National Institute for Health and Care Excellence (NICE) decision support unit recently produced a technical support document that included a discussion of conceptualizing economic models [59]. In developing the new CM of COPD presented in this paper, the principles of good modeling practice laid out in the SMDM/ISPOR Task Force 2 paper were followed as far as possible. The only part of this guidance that proved challenging to implement was II-3, which states that "although data are essential to a model, the conceptual structure should be driven by the decision problem or research question and not determined by data availability." In the present study, when moving from draft to final CM, considerations about data availability from the ECLIPSE study were important. The CM should be refined as additional data become available.

In the NICE decision support unit report a distinction is made between 'problem-orientated conceptual modeling' and 'design-orientated conceptual modeling' [58]. Based on the present study, we suggest that the first step is 'problem-orientated' conceptual modeling, which involves deciding how the complexity of the 'real world' can be represented in a simplified modeling form. The CM may then be refined into a 'design-orientated' CM that takes into consideration how the model will be implemented in practice. During this step, it is legitimate to consider 
data availability and how the parameters of the final model are to be estimated. While we agree with the principle listed in the SMDM/ISPOR Task Force Report, this sets a very high bar. In practice, the distinction between two forms of conceptual modeling made in the NICE decision support unit report was reflected in our experience of constructing a new model for COPD. We acknowledge that a draft model of potential associations for which there are no data would guide future exploration rather than limiting to a CM that is already modified for available data; this model would ensure that all relevant aspects of a disease are considered.

\section{CONCLUSIONS}

We have developed and quantified a CM that reflects the heterogeneous nature of COPD, which includes a wide variety of COPD attributes impacting disease progression and health outcomes. The CM was refined using available evidence to validate the impact of the selected attributes on economic, humanistic and clinical outcomes. This CM is the foundation for the development of a new COPD disease progression model based on the ECLIPSE database. It has been formulated in a way that will facilitate its implementation in future disease progression and economic models, by serving as a framework for the analysis of patient-level data. The CM has applications both in clinical decision making as well as in economic modeling and provides an example for the development of CMs in other therapy areas for use in disease progression modeling. Future research should focus on the estimation of associations to incorporate the attributes in COPD that have been excluded from this $\mathrm{CM}$, in order to understand the implications of their exclusion on the accuracy of the representation of disease progression and management, and thereby the usefulness of the model. Additional research may still be required in order to understand these associations.

\section{ACKNOWLEDGMENTS}


The authors acknowledge the contributions of members of the Delphi Panel: Roberto dal Negro MD, FCCP (Lung Department, Orlandi General Hospital, Verona, Italy), Michael Polkey PhD (Professor of Respiratory Medicine, Imperial College London, UK), Doug Mapel MD, MPH, FACCP (Medical Director of the Lovelace Clinic Foundation, Arizona, USA), David Mannino MD FCCP (Professor, Department of Preventive Medicine and Environmental Health, USA), Tobias Welte MD, PhD (Professor of Pulmonary Medicine, Hannover Medical School, Germany) and acknowledge Afisi S. Ismaila PhD (Value Evidence and Outcomes, GSK R\&D, Research Triangle Park, North Carolina, USA and Department of Clinical Epidemiology and Biostatistics, McMaster University, Hamilton, Ontario, Canada) for his scientific contribution, preparation and technical editing of the manuscript.

Editorial support in the form of editorial suggestions to draft versions of this paper, assembling tables and figures, collating author comments, copyediting, fact checking, referencing and graphic services was provided by David Cutler, PhD and Laura Maguire, MChem at Gardiner-Caldwell Communications (Macclesfield, UK) and was funded by GSK.

\section{AUTHOR CONTRIBUTIONS}

MT assisted in design of the literature review, drafted the initial conceptual model for review, developed and conducted the Delphi Panel exercise, participated in finalizing the conceptual model, led writing of the manuscript and approved the manuscript for submission.

SGM conducted the literature review, drafted the initial conceptual model for review, assisted in the Delphi Panel exercise, analyzed the Delphi Panel results, participated in finalizing the conceptual model, quantified the conceptual model, led writing of sections of the manuscript and approved the manuscript for submission.

HM drafted the initial conceptual model for review, participated in finalizing the conceptual model and critically reviewed and approved the manuscript. 
AB drafted the initial conceptual model for review, participated in finalizing the conceptual model, quantified the conceptual model and critically reviewed and approved the manuscript.

MRvM participated in finalizing the conceptual model and critically reviewed and approved the manuscript.

MC drafted the initial conceptual model for review, assisted in the literature review and Delphi Panel exercise, participated in finalizing the conceptual model and critically reviewed and approved the manuscript.

DAL participated in finalizing the conceptual model and critically reviewed and approved the manuscript.

\section{AUTHOR CONFLICTS OF INTEREST}

MT, HM, MC are employees of and hold stock in GSK.

SGM was an employee of GSK at the time of the research and analyses of this project, and is currently an employee of Amgen.

AB has received funding as a consultant and through Oxford Outcomes Ltd from GSK related to the development of this model.

MRvM has published on other economic models of chronic obstructive pulmonary disease, either developed with public funding or pharmaceutical industry funding. She has received a fee for participating in the Steering Group. In recent years she has received unrestricted research grants from Astellas (Nycomed/Takeda), Boehringer Ingelheim, and GSK. She also received speaker's fees from these companies.

DAL has received grant support, honoraria and consultancy fees from GSK. He is the Chair of the GSK Respiratory Therapy Area Board. 


\section{REFERENCES}

1. Agustí AG, Noguera A, Sauleda J, Sala E, Pons J, Busquets X. Systemic effects of chronic obstructive pulmonary disease. Eur Respir J. 2003 Feb;21:347-60.

2. Roberts M, Russell LB, Paltiel AD, Chambers M, McEwan P, Krahn M; ISPOR-SMDM Modeling Good Research Practices Task Force. Conceptualizing a model: a report of the ISPOR-SMDM Modeling Good Research Practices Task Force-2. Value Health. 2012 Sep-Oct;15:804-11.

3. Bowen GA. Naturalistic inquiry and the saturation concept: a research note. Qual Res. 2008 Feb;8:137-52.

4. Guest G, Bunce A, Johnson L. How many interviews are enough? An experiment with data saturation and variability. Field Methods 2006 Feb;18:59-82.

5. Earp JA, Ennett ST. Conceptual models for health education research and practice. Health Educ Res. 1991 Jun;6:163-71.

6. Vestbo J, Anderson W, Coxson HO, Crim C, Dawber F, Edwards L, et al; ECLIPSE investigators. Evaluation of COPD Longitudinally to Identify Predictive Surrogate End-points (ECLIPSE). Eur Respir J. 2008 Apr;31:869-7.

7. Agusti A, Calverley PM, Celli B, Coxson HO, Edwards LD, Lomas DA, et al; Evaluation of COPD Longitudinally to Identify Predictive Surrogate Endpoints (ECLIPSE) investigators. Characterisation of COPD heterogeneity in the ECLIPSE cohort. Respir Res. 2010 Sep 10;11:122.

8. Borson S, Scanlan J, Friedman S, Zuhr E, Fields J, Aylward E, et al. Modeling the impact of COPD on the brain. Int J Chron Obstruct Pulmon Dis 2008;3:429-34.

9. Cote C, Zilberberg MD, Mody SH, Dordelly LJ, Celli B. Haemoglobin level and its clinical impact in a cohort of patients with COPD. Eur Respir J. 2007 May;29:923-9.

10. Daudey L, Peters JB, Molema J, Dekhuijzen PN, Prins JB, Heijdra YF, et al. Health status in COPD cannot be measured by the St George's Respiratory Questionnaire alone: an evaluation of the underlying concepts of this questionnaire. Respir Res. 2010 Jul 22;11:98. 
11. Eisner MD, Blanc PD, Yelin EH, Katz PP, Sanchez G, Iribarren C, et al. Influence of anxiety on health outcomes in COPD. Thorax. 2010 Mar;65:229-34.

12. Ferrari R, Tanni SE, Lucheta PA, Faganello MM, do Amaral RA, Godoy I. Gender differences in predictors of health status in patients with COPD. J Bras Pneumol. 2010 Jan-Feb;36:37-43.

13. Giardino ND, Curtis JL, Andrei A, Fan VS, Benditt JO, Lyubkin M, et al; NETT Research Group. Anxiety is associated with diminished exercise performance and quality of life in severe emphysema: a crosssectional study. Respir Res. 2010 Mar 9;11:29.

14. Jones PW. Activity limitation and quality of life in COPD. COPD. 2007 Sep;4:273-8.

15. Jones RC, Donaldson GC, Chavannes NH, Kida K, Dickson-Spillmann M, Harding S, et al. Derivation and validation of a composite index of severity in chronic obstructive pulmonary disease: the DOSE index. Am J Respir Crit Care Med. 2009 Dec 15;180:1189-95.

16. Jones PW, Donohue J, Nedelman J, Pinault G, Pascoe S. Correlating changes in lung function with patient reported outcomes in COPD. Thorax. 2010;65(Suppl 4):A141.

17. Lemmens KM, Nieboer AP, Huijsman R. Designing patient-related interventions in COPD care: empirical test of a theoretical model. Patient Educ Couns. 2008 Aug;72:223-31.

18. Meguro M, Barley EA, Spencer S, Jones PW. Development and validation of an improved, COPD-specific version of the St. George respiratory questionnaire. Chest. 2007 Aug;132:456-63.

19. Pirraglia PA, Casserly B, Velasco R, Borgia ML, Nici L. Association of change in depression and anxiety symptoms with functional outcomes in pulmonary rehabilitation patients. J Psychosom Res. 2011 Jul;71:45-9.

20. Probst-Hensch NM, Curjuric I, Pierre-Olivier B, Ackermann-Liebrich U, Bettschart RW, Brändli O, et al. Longitudinal change of prebronchodilator spirometric obstruction and health outcomes: results from the SAPALDIA cohort. Thorax. 2010 Feb;65:150-6.

21. Singh D, Edwards L, Tal-Singer R, Rennard S. Sputum neutrophils as a biomarker in COPD: findings from the ECLIPSE study. Respir Res. 2010 Jun 15;77. 
22. Vercoulen JH, Daudey L, Molema J. An integral assessment framework of health status in chronic obstructive pulmonary disease. Int J Behav Med. 2008;15:263-79.

23. Verhage TL, Heijdra YF, Molema J, Daudey L, Dekhuijzen PN, Vercoulen JH. Adequate patient characterization in COPD: reasons to go beyond GOLD classification. Open Respir Med J. 2009 Feb $13 ; 3: 1-9$.

24. Victorson DE, Anton S, Hamilton A, Yount S, Cella D. A conceptual model of the experience of dyspnea and functional limitations in chronic obstructive pulmonary disease. Value Health. 2009 Sep;12:1018-25.

25. Dodd JW, Getov SV, Jones PW. Cognitive function in COPD. Eur Respir J. 2010 Apr;35:913-22.

26. Stull DE, Leidy NK, Jones PW, Ståhl E. Measuring functional performance in patients with COPD: a discussion of patient-reported outcome measures. Curr Med Res Opin. 2007 Nov;23:2655-65.

27. Benzo RP, Chang CC, Farrell MH, Kaplan R, Ries A, Martinez FJ, et al; NETT Research Group. Physical activity, health status and risk of hospitalization in patients with severe chronic obstructive pulmonary disease. Respiration. 2010;80:10-8.

28. Yentes JM, Sayles H, Meza J, Mannino DM, Rennard SI, Stergiou N. Walking abnormalities are associated with COPD: an investigation of the NHANES III dataset. Respir Med. 2011 Jan;105:1:80-7.

29. Zaniolo O, Iannazzo S, Pradelli L, Miravitlles M. Pharmacoeconomic evaluation of tiotropium bromide in the long-term treatment of chronic obstructive pulmonary disease (COPD) in Italy. Eur J Health Econ. 2012 Feb;13:71-80.

30. Man SF, Xing L, Connett JE, Anthonisen NR, Wise RA, Tashkin DP, et al. Circulating fibronectin to Creactive protein ratio and mortality: a biomarker in COPD? Eur Respir J. 2008 Dec;32:1451-7.

31. Peruzza S, Sergi G, Vianello A, Pisent C, Tiozzo F, Manzan A, et al. Chronic obstructive pulmonary disease (COPD) in elderly subjects: impact on functional status and quality of life. Respir Med. 2003 Jun;97:612-7.

32. Briggs A, Spencer M, Wang H, Mannino D, Sin DD. Development and validation of a prognostic index for health outcomes in chronic obstructive pulmonary disease. Arch Intern Med. 2008 Jan 14;168:71-9. 
33. Curkendall SM, Lanes S, De Luise C, Stang MR, Jones JK, She D, et al. Chronic obstructive pulmonary disease severity and cardiovascular outcomes. Eur J Epidemiol. 2006;21:803-13.

34. De Voogd JN, Wempe JB, Koeter GH, Postema K, van Sonderen E, Ranchor AV, et al. Depressive symptoms as predictors of mortality in patients with COPD. Chest. 2009 Mar;135:619-25.

35. Halpin DM, Peterson S, Larsson TP, Calverley PM. Identifying COPD patients at increased risk of mortality: predictive value of clinical study baseline data. Respir Med. 2008 Nov;102:1615-24.

36. Malo De Molina R, Mortensen EM, Restrepo MI, Copeland LA, Pugh MJ, Anzueto A. Inhaled corticosteroid use is associated with lower mortality for subjects with COPD and hospitalised with pneumonia. Eur Respir J. 2010 Oct;36:751-7.

37. Oga T, Nishimura K, Tsukino M, Sato S, Hajiro T, Ikeda A, et al. Health status measured with the CRQ does not predict mortality in COPD. Eur Respir J. 2002 Nov;20:1147-51.

38. Oga T, Nishimura K, Tsukino M, Sato S, Hajiro T. Analysis of the factors related to mortality in chronic obstructive pulmonary disease: role of exercise capacity and health status. Am J Respir Crit Care Med. 2003 Feb 15;167:544-9.

39. Rashid M, Brown J, Ling HZ, Aggarwal S, Flint J, Mendonca M, et al. Expansion of the red cell distribution width and evolving iron deficiency as predictors of poor outcome in chronic obstructive pulmonary disease. Thorax. 2010;65(Suppl 4):A75-6.

40. Schembri S, Anderson W, Morant S, Winter J, Thompson P, Pettitt D, et al. A predictive model of hospitalisation and death from chronic obstructive pulmonary disease. Respir Med. 2009 Oct;103:1461-7.

41. Stavem K, Sandvik L, Erikssen J. Can global initiative for chronic obstructive lung disease stage 0 provide prognostic information on long-term mortality in men? Chest. 2006 Aug;130:318-25.

42. Vaz Fragoso CA, Concato J, McAvay G, Van Ness PH, Rochester CL, Yaggi HK, et al. The ratio of FEV1 to FVC as a basis for establishing chronic obstructive pulmonary disease. Am J Respir Crit Care Med. 2010 Mar 1;181:446-51. 
43. Yohannes AM, Baldwin RC, Connolly MJ. Predictors of 1-year mortality in patients discharged from hospital following acute exacerbation of chronic obstructive pulmonary disease. Age Ageing 2005 Sep;34:5:491-6.

44. Aaron SD, Ramsay T, Vandemheen K, Whitmore GA. A threshold regression model for recurrent exacerbations in chronic obstructive pulmonary disease. J Clin Epidemiol. 2010 Dec;63:1324-31.

45. Fan VS, Ramsey SD, Make BJ, Martinez FJ. Physiologic variables and functional status independently predict COPD hospitalizations and emergency department visits in patients with severe COPD. COPD. 2007 Mar;4:29-39.

46. Garcia-Aymerich J, Farrero E, Felez MA, Izquierdo J, Marrades RM, Antó JM; Estudi del Factors de Risc d'Agudització de la MPOC investigators. Risk factors of readmission to hospital for a COPD exacerbation: a prospective study. Thorax. 2003 Feb;58:100-5.

47. Niewoehner DE, Lokhnygina Y, Rice K, Kuschner WG, Sharafkhaneh A, Sarosi GA, et al. Risk indexes for exacerbations and hospitalizations due to COPD. Chest. 2007 Jan;131:20-8.

48. Stolz D, Breidthardt T, Christ-Crain M, Bingisser R, Miedinger D, Leuppi J, et al. Use of B-type natriuretic peptide in the risk stratification of acute exacerbations of COPD. Chest. 2008 May;133:1088-94.

49. Suh DC, Lau H, La HO, Choi IS, Geba GP. Association between incidence of acute exacerbation and medication therapy in patients with COPD. Curr Med Res Opin. 2010 Feb;26:297-306.

50. Fan VS, Bridevaux PO, McDonell MB, Fihn SD, Besser LM, Au DH. Regional variation in health status among chronic obstructive pulmonary disease patients. Respiration. 2011;81:9-17.

51. Crisafulli E, Costi S, Luppi F, Cirelli G, Cilione C, Coletti O, et al. Role of comorbidities in a cohort of patients with COPD undergoing pulmonary rehabilitation. Thorax. 2008 Jun;63:487-92.

52. Casaburi R, Porszasz J, Burns MR, Carithers ER, Chang RS, Cooper CB. Physiologic benefits of exercise training in rehabilitation of patients with severe chronic obstructive pulmonary disease. Am J Respir Crit Care Med. 1997 May;155:1541-51.

53. Donaldson GC, Müllerova H, Locantore N, Hurst JR, Calverley PM, Vestbo J, et al. Factors associated with change in exacerbation frequency in COPD. Respir Res. 2013 Jul 30;14:79. 
54. Celli B, Clifford S, Kawata A, Casaburi R, Criner GJ, Dangeti R, et al. The 6-minute walk test (6MWD) can stratify COPD patients at risk of death, hospitalization and exacerbations. Am J Respir Crit Care Med. 2014;189:A5857.

55. Global Initiative for Chronic Obstructive Lung Disease. Global Strategy for Diagnosis, Management, and Prevention of COPD. Updated January 2014. http://www.goldcopd.org/. Accessed 13 February 2015.

56. Singh SJ, Puhan MA, Andrianopoulos V, Hernandes NA, Mitchell KE, Hill CJ, et al. An official systematic review of the European Respiratory Society/American Thoracic Society: Measurement properties of field walking tests in chronic respiratory disease. Eur Respir J. 2014;44:1447-78.

57. Spruit MA, Watkins ML, Edwards LD, Vestbo J, Calverley PM, Pinto-Plata V, et al; Evaluation of COPD Longitudinally to Identify Predictive Surrogate Endpoints (ECLIPSE) study investigators. Determinants of poor 6-min walking distance in patients with COPD: the ECLIPSE cohort. Respir Med. 2010 Jun;104:84957.

58. Kaltenthaler E, Tappenden P, Paisley S, Squires H. NICE DSU Technical Support Document 13: identifying and reviewing evidence to inform the conceptualisation and population of cost effectiveness models. 2011. http://www.nicedsu.org.uk/TSD\%2013\%20model\%20parameters.pdf. Accessed 13 February 2015 .

59. Calverley PM, Anderson JA, Celli B, Ferguson GT, Jenkins C, Jones PW, et al; TORCH investigators. Salmeterol and fluticasone propionate and survival in chronic obstructive pulmonary disease. N Engl J Med. 2007 Feb 22;356:775-89. 
Table 1 Statistical Significance of Pairwise Correlations Between Explanatory (Baseline) Variables and Dependent (Disease Progression Attributes) Variables

Based on ECLIPSE Data

\begin{tabular}{|c|c|c|c|c|c|c|c|c|c|}
\hline \multirow{15}{*}{ 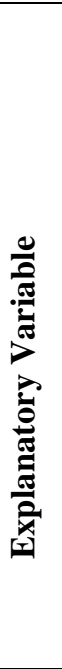 } & & \multicolumn{8}{|c|}{ Dependent Variable } \\
\hline & & BMI & $\begin{array}{c}\text { CVD/ } \\
\text { Comorbidities }\end{array}$ & Depression & Exacerbation & Exercise & Fibrinogen & Lung function & $\begin{array}{c}\text { Signs and } \\
\text { Symptoms }\end{array}$ \\
\hline & BMI & & $<0.0001$ & & & $<0.0001$ & $<0.0001$ & & \\
\hline & CRP & $<0.0001$ & 0.002 & & & & & & \\
\hline & CVD/Comorbidities & $<0.0001$ & & & 0.32 & $<0.0001$ & $<0.0001$ & $<0.0001$ & $<0.0001$ \\
\hline & Depression & & & & 0.0012 & & & & \\
\hline & Exacerbation & & 0.46 & 0.0012 & & $<0.0001$ & $<0.0001$ & $<0.0001$ & $<0.0001$ \\
\hline & Exercise & $<0.001$ & $<0.0001$ & & $<0.0001$ & & & $<0.0001$ & $<0.0001$ \\
\hline & Fibrinogen & $<0.001$ & $<0.0001$ & & $<0.0001$ & & & & \\
\hline & Gender & & & & & & 0.90 & & \\
\hline & Lung function & & $<0.0001$ & & $<0.0001$ & $<0.0001$ & & & $<0.0001$ \\
\hline & Mortality & 0.0016 & $<0.0001$ & 0.96 & 0.002 & $<0.0001$ & & $<0.0001$ & $<0.0001$ \\
\hline & HRQoL & $<0.0001$ & $<0.0001$ & $<0.0001$ & $<0.0001$ & $<0.0001$ & & $<0.0001$ & $<0.0001$ \\
\hline & Signs and Symptoms & & $<0.0001$ & & $<0.0001$ & $<0.0001$ & & $<0.0001$ & \\
\hline & Smoking & & & 0.054 & 0.16 & $<0.0001$ & 0.008 & $<0.0001$ & 0.0009 \\
\hline
\end{tabular}

BMI, body mass index; CRP, C-reactive protein; CVD, cardiovascular disease; HRQoL, health-related quality of life. 


\section{Figure legends}

Figure 1. Results of literature search.

COPD, chronic obstructive pulmonary disease.

Figure 2. Reporting of COPD attributes as dependent or explanatory variables from the literature review.

Values give the number of reports of an association of a particular disease attribute with another attribute, or with a COPD health outcomes. Source studies are likely to have provided many associations (for different attributes).

COPD, chronic obstructive pulmonary disease; HRQoL, heath-related quality of life.

Figure 3. List of (a) symptoms and (b) attributes associated with exercise, identified in the literature review.

Values give the number of reports of the association with symptoms or other attributes.

HRQoL, heath-related quality of life. *A subjective complaint is independent of a measurable symptom (i.e., patient complaint or feeling of impairment based on their own perception, without an actual measure of either).

Figure 4. (a) Draft conceptual model of COPD (proposed by the Steering Group) and (b) final conceptual model.

COPD, chronic obstructive pulmonary disease; BMI, body mass index; CVD, cardiovascular disease; HRQLL, health-related quality of life. 
Figure 1. Results of literature search.

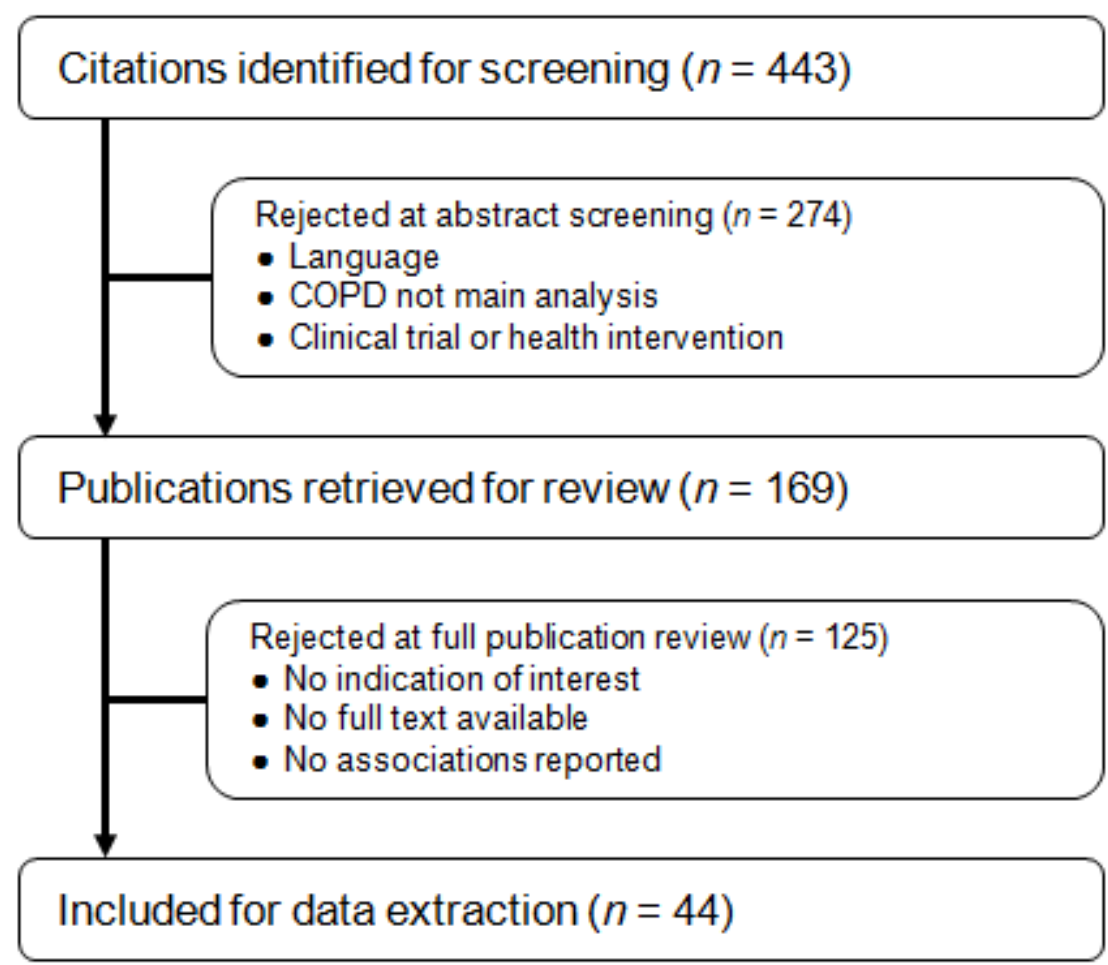

COPD, chronic obstructive pulmonary disease. 
Figure 2. Reporting of COPD attributes as dependent or explanatory variables from the literature review.

Values give the number of reports of an association of a particular disease attribute with another attribute, or with a COPD health outcomes. Source studies are likely to have provided many associations (for different attributes).

COPD, chronic obstructive pulmonary disease; HRQoL, heath-related quality of life.

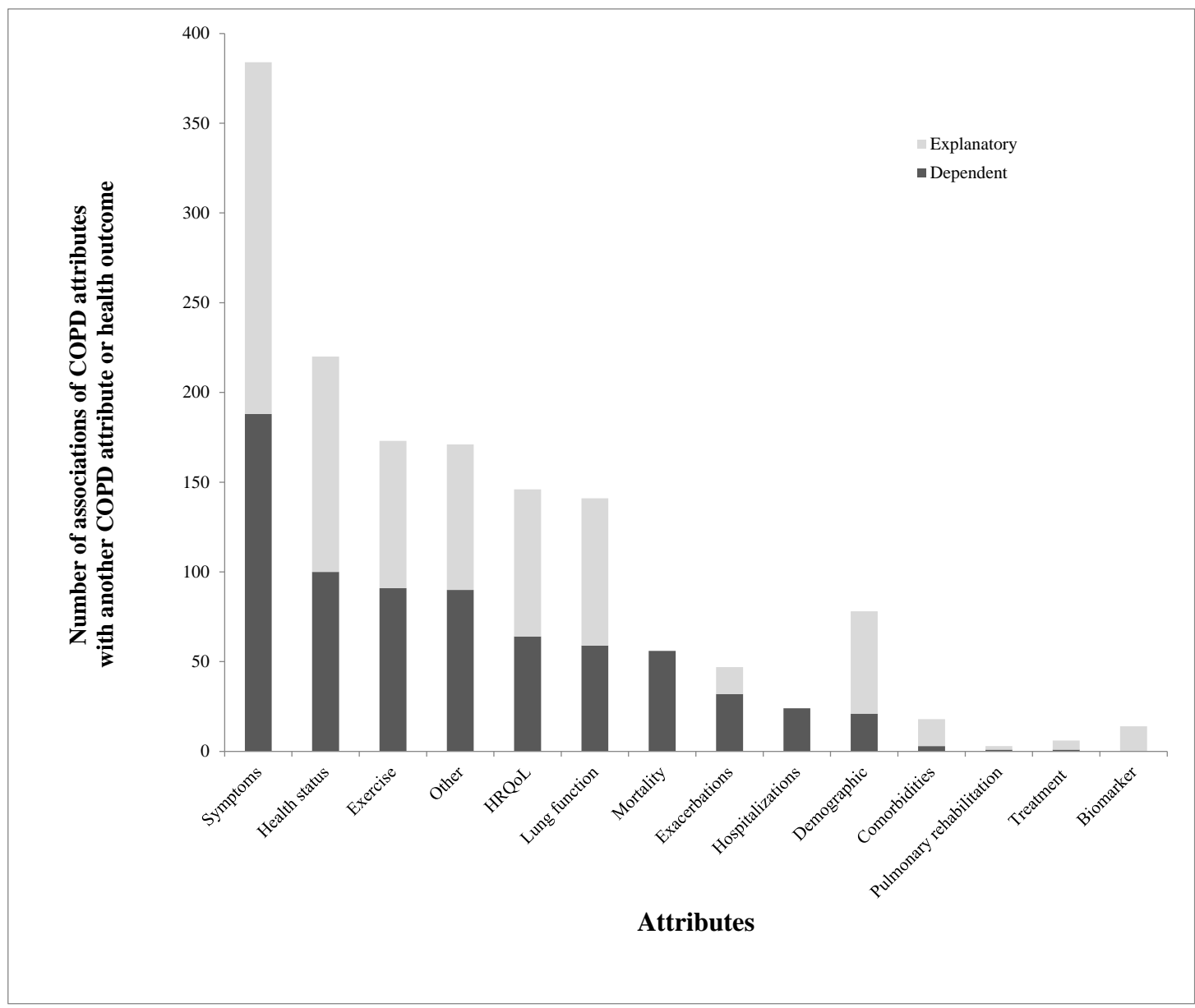

Total no. of reported associations $=730$.

Dependent variables: Symptoms: [8-24]; health status: [11,13-19,21,24,26]; exercise: [8-15,17,18,2225,27,28]; other: [10,12,17,25,29]; HRQoL: [10,11,13,17,18,22,23]; lung function: [8,11,12,15,17,18,2123,30,31]; mortality: [9,25,30,32-43]; exacerbations: [11,14,16,21,32,44-47]; hospitalizations:

[25,27,32,33,40,47,48]; demographic: [12,15,22-24,30,49]; comorbidities: [8,49,50]; pulmonary rehabilitation: [51]; treatment: [49]. 
Explanatory variables: Symptoms: [11,13-15,17,19,22,24,25,27,28,31,34,35,37,40,43,45]; health status: [8,10,15,17,18,26-28,31,32,35,37,38,41,43,45,47]; exercise: [8,13,14,17,22,24,27,28,31,45,46]; lung function: [8,9,15-17,20,22,23,25,27,32,34,35,37,38,40,42,45,46,47]; other: [17,25]; HRQoL: [17,22,32,38,43,50]; demographic: [9,12,15,22,28,32,34,35,37,38,40,47]; comorbidities: [8,9,25,32,33,40,45,47,51]; exacerbations: [15,32,44-47,49]; biomarker: [9,21,30,39,48]; treatment: [8,29,36,43]; pulmonary rehabilitation: [19,25].

COPD, chronic obstructive pulmonary disease; HRQoL, heath-related quality of life. 
Figure 3. List of (a) symptoms and (b) attributes associated with exercise, identified in the literature review.

Values give the number of reports of the association with symptoms or other attributes.

HRQoL, heath-related quality of life. *A subjective complaint is independent of a measurable symptom (i.e., patient complaint or feeling of impairment based on their own perception, without an actual measure of either).

(a)

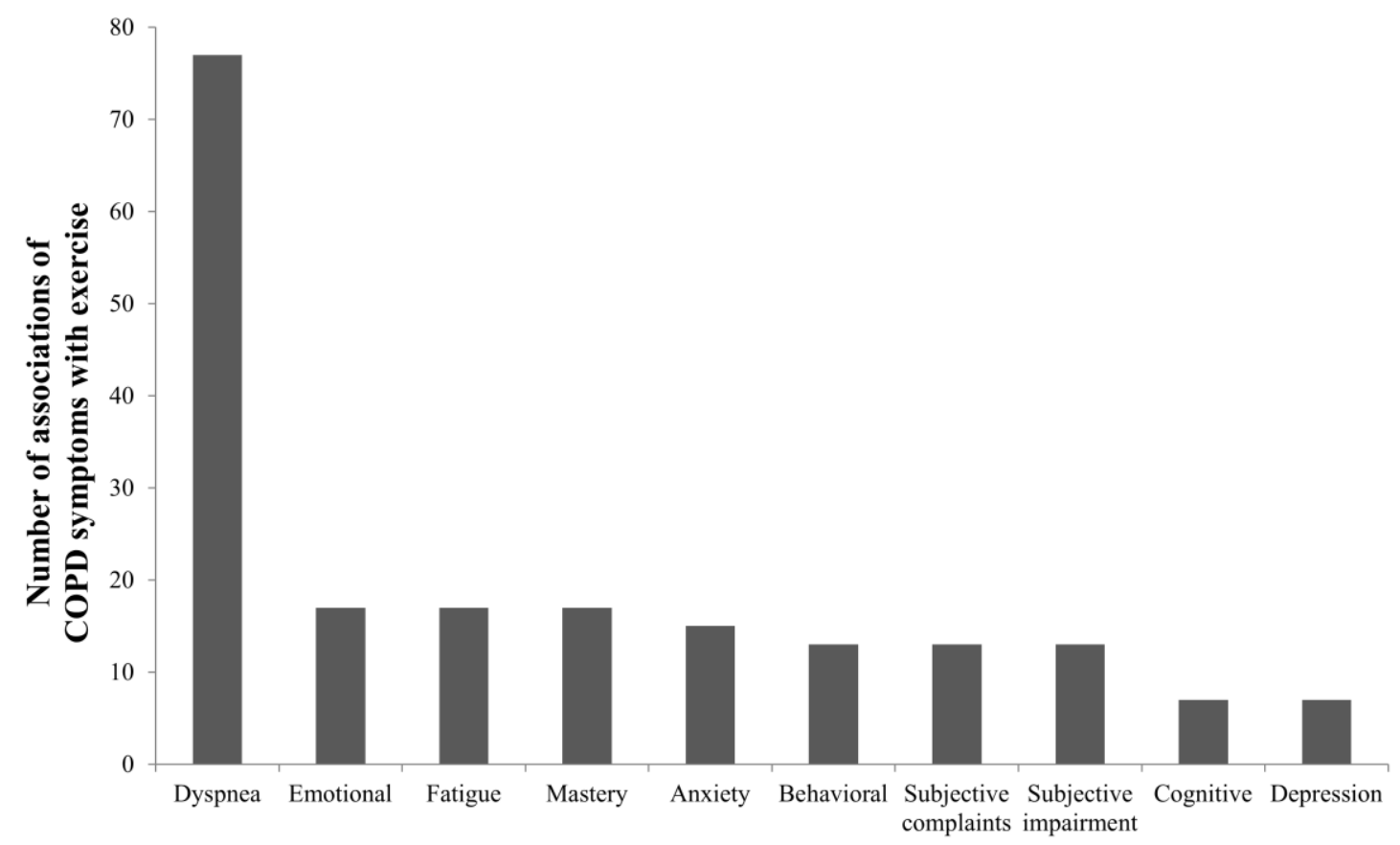

Attributes

Total no. of reported symptoms $=196$. Dyspnea: $[14,15,17,22,24,31,34,38,40,43]$; emotional: [27]; fatigue:

[45]; mastery: [22]; anxiety: [11,13,19,27]; behavioral: [22]; subjective complaints: [22]; subjective impairment: [17,28]; cognitive: [25,35]; depression: [19,25,38,43]. 
(b)

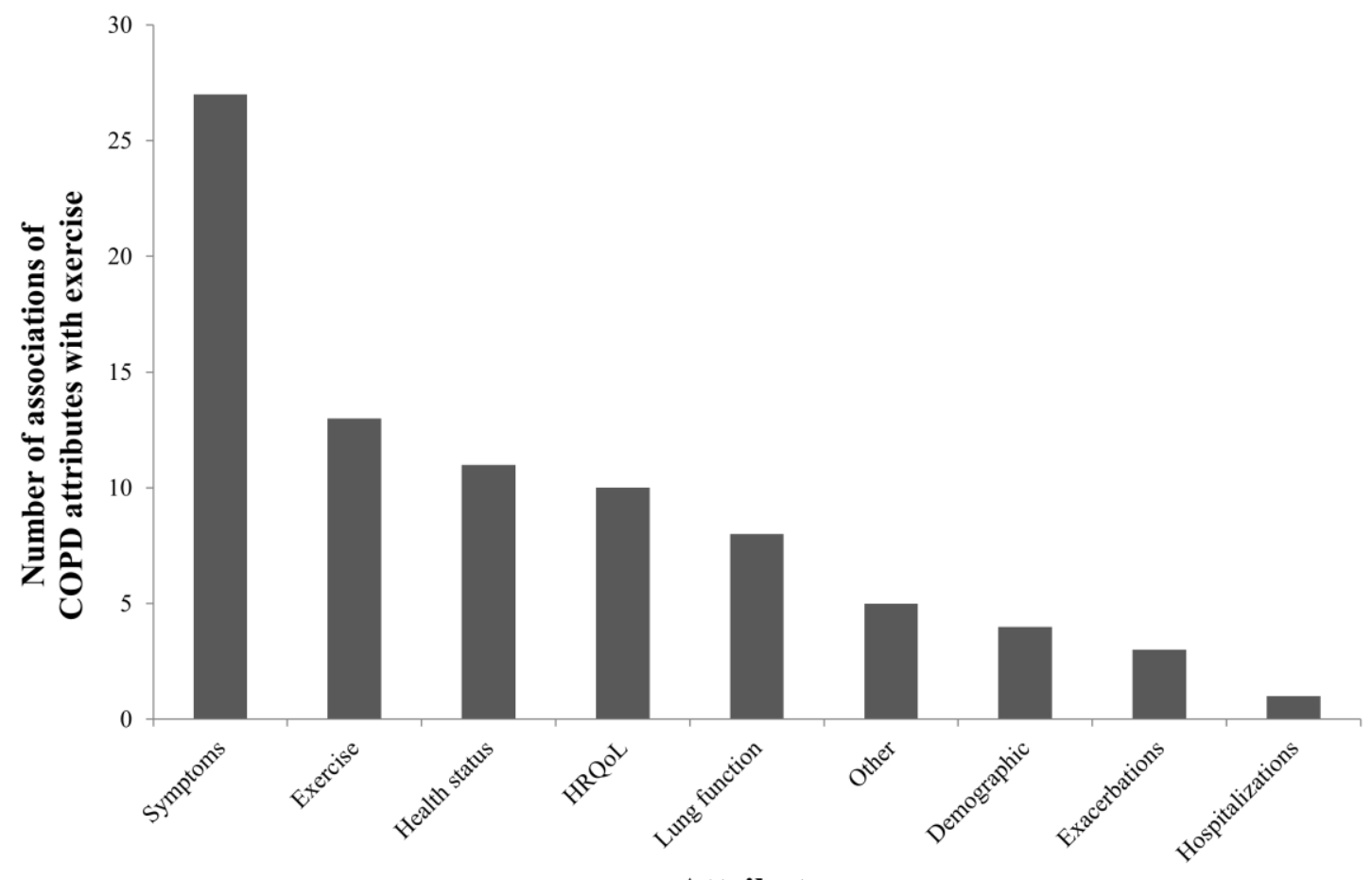

Attributes

Total no. of reported associations $=82$. Symptoms: $[8,13,14,17,22,24]$; exercise: $[8,22,24,27,28]$; health status: [13,14,17,24]; HRQoL: [17,22]; lung function: [17,22,31]; other: [17]; demographic: [22,24]; exacerbations: [45,46]; hospitalizations: [27].

HRQoL, heath-related quality of life. *A subjective complaint is independent of a measurable symptom (i.e., patient complaint or feeling of impairment based on their own perception, without an actual measure of either). 
Figure 4. (a) Draft conceptual model of COPD (proposed by the Steering Group) and (b) final conceptual model.

(a)

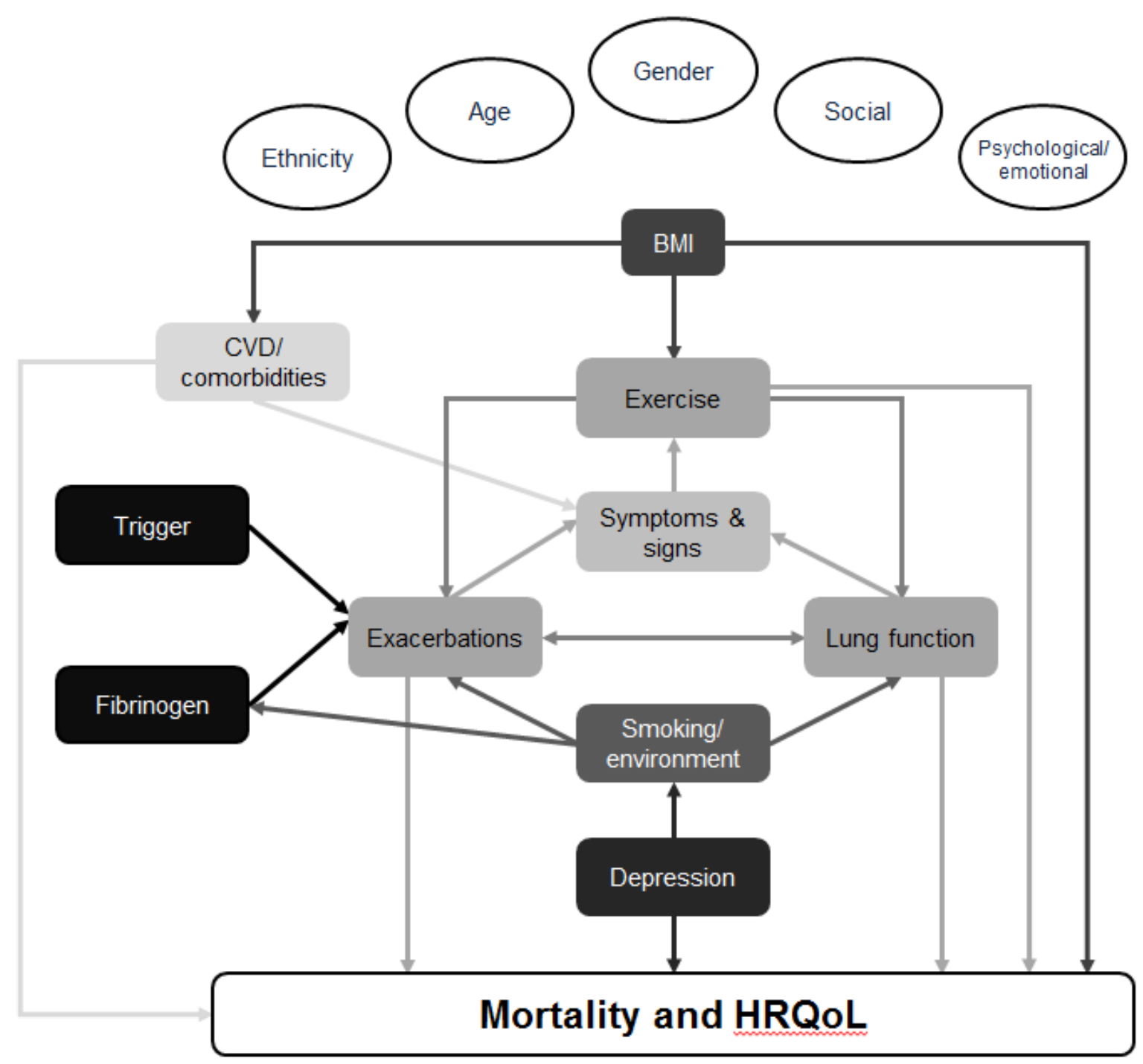


(b)

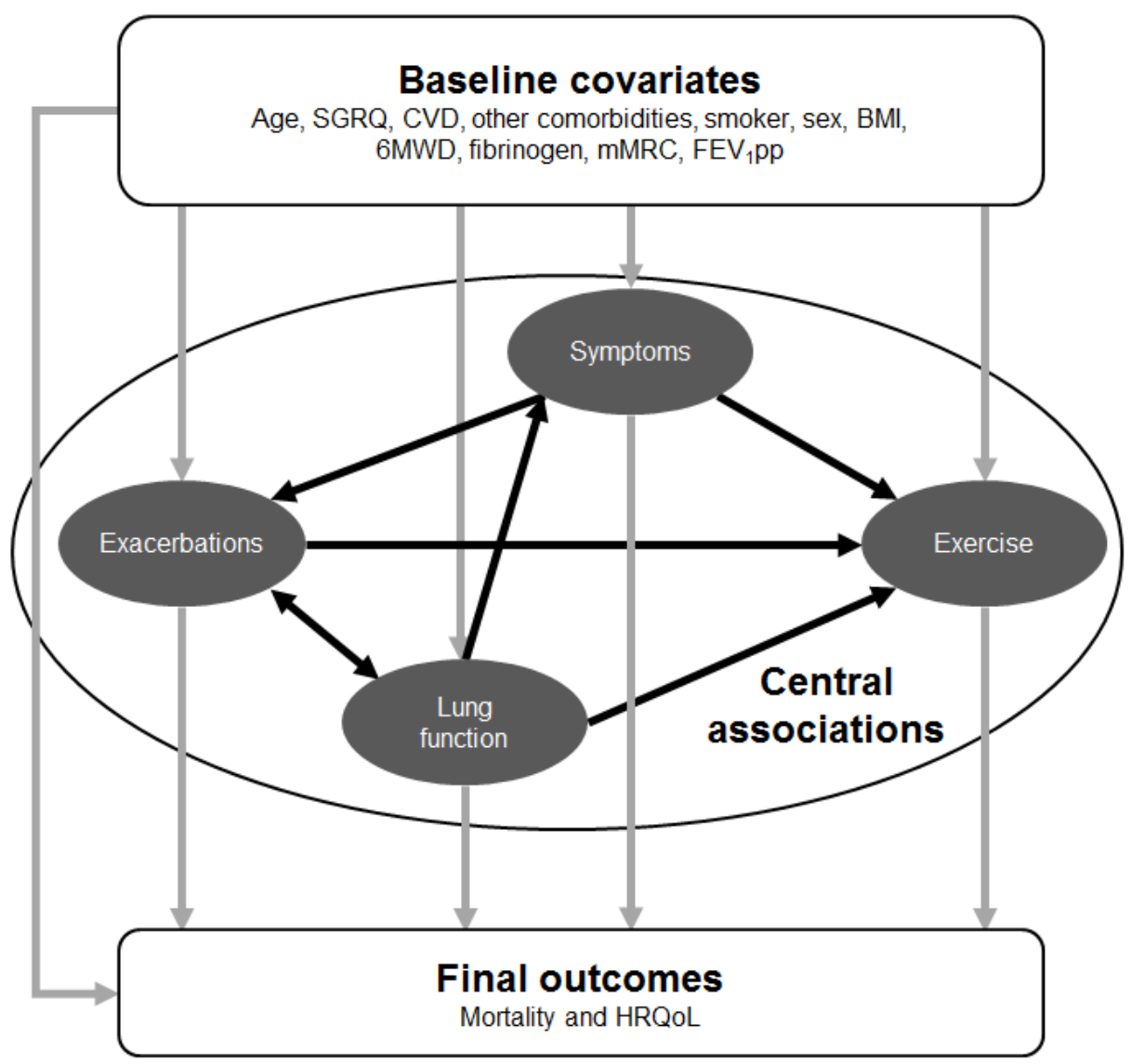

6MWD, 6-minute walk test distance; BMI, body mass index; COPD, chronic obstructive pulmonary disease;

CVD, cardiovascular disease; $F E V_{l} p p$, forced expiratory volume in 1 second percent predicted; HRQoL,

health-related quality of life; mMRC, modified Medical Research Council dyspnea scale; SGRQ, St-George's Respiratory Questionnaire. Note: Final outcomes are influenced both by baseline covariates and attributes of COPD which change over time, as described in the central section of the model. 